\title{
EXISTENCE OF GLOBAL ATTRACTOR FOR CAUCHY PROBLEMS
}

\author{
Sung Kyu Choi*, Hyun Ho Jang**, Namjip Koo***, \\ AND Chanmi Yun****
}

\begin{abstract}
We investigate the existence of a global attractor for the Cauchy problem

$$
x^{\prime}(t)=A x(t)+F(x(t)), x(0)=x_{0} \in X
$$

on a Banach space $X$ according to the remark in You and Yuan's paper.
\end{abstract}

\section{Introduction}

The theory of global attractor is one of important object to describe long time dynamics of an infinite dimensional system. It is very useful to investigate the asymptotic behavior since it is valid for more general situations than those for stability.

Caraballo et al. [5] proved the existence of attractors for

$$
\begin{cases}x^{\prime}(t) & =f\left(t, x_{t}\right), t>\tau \\ x_{\tau} & =\phi \in C_{\gamma},\end{cases}
$$

where $C_{\gamma}$ is the Banach space defined by

$$
C_{\gamma}=\left\{x \in C\left((-\infty, 0], \mathbb{R}^{n}\right): \exists \lim _{\theta \rightarrow-\infty} e^{\gamma \theta} x(\theta)\right\},
$$

with the norm

$$
\|x\|=\sup _{-\infty<\theta \leq 0} e^{\gamma \theta}|x(\theta)|, \gamma>0
$$

Received June 01, 2013; Accepted July 19, 2013.

2010 Mathematics Subject Classification: Primary 35B41, 34K30, 35B40.

Key words and phrases: global attractor, integral solution, generator, point dissipative, functional differential equation, Hille-Yosida operator.

Correspondence should be addressed to Namjip Koo, njkoo@cnu.ac.kr.

This work was supported by Basic Science Research Program through the National Research Foundation of Korea(NRF) funded by the Ministry of Education(NRF2013R1A1A2007585). 
and $C\left((\infty, 0], \mathbb{R}^{n}\right)$ is the space of continuous functions from $(-\infty, 0]$ to $\mathbb{R}^{n}$ with the sup-norm

$$
\|\phi\|=\sup _{-\infty<s \leq 0}|\phi(s)|,
$$

and define $x_{t} \in C\left((-\infty, 0], \mathbb{R}^{n}\right)$ by $x_{t}(s)=x(t+s), s \in(-\infty, 0]$, for a function $x$.

Zelatti [12] studied the existence of a global attractor under minimal requirements in terms of continuity of the multivalued semigroup of operators acting on complete metric spaces.

Bouzahir and Ezzinbi [3] investigated the existence of a global attractor for the equation

$$
\begin{cases}x^{\prime}(t) & =A x(t)+F\left(x_{t}\right), t \geq 0, \\ x_{0} & =\phi \in B\end{cases}
$$

where $A: D(A) \subset X \rightarrow X$ is a closed linear operator on a Banach space $X$ and $B$ is the phase space by Hale and Kato [7]. Also, Bouzahir et al. studied the existence of a global attractor for (1.2) under the initial condition $x_{0}=\varphi \in C_{\gamma}$ in [2]. You and Yuan [11] investigated the existence of a global attractor for (1.2) under the initial condition $x_{0}=\varphi \in C=C([-r, 0], X), r>0$.

In this paper, we will prove the existence of a global attractor for the equation without delay $(r=0)$

$$
\left\{\begin{array}{l}
x^{\prime}(t)=A x(t)+F(x(t)), t \geq 0, \\
x(0)=x_{0} \in X
\end{array}\right.
$$

according to Remark 3.3 in [11].

\section{Existence of global attractor}

We consider the partial functional differential equation

$$
\begin{cases}x^{\prime}(t) & =A x(t)+F\left(x_{t}\right), t \geq 0, \\ x_{0} & =\phi \in C=C([-r, 0], X), r>0 .\end{cases}
$$

Here $C([-r, 0], X)$ is the space of continuous functions from $[-r, 0]$ into a Banach space $X$. We assume that the following.

(H1) The linear operator $A$ is a Hille-Yosida operator, that is, there exist $M \geq 1$ and $\omega \in \mathbb{R}$ such that

(i) $(\omega, \infty) \subset \rho(A)$

(ii) $\left\|(\lambda I-A)^{-n}\right\| \leq \frac{M}{(\lambda-\omega)^{n}}$ for $\lambda>\omega$, 
where $\rho(A)$ is the resolvent set of $A$.

(H2) $F: C \rightarrow X$ is Lipschitz continuous, i.e., there exists $L>0$ such that

$$
\left\|F\left(\phi_{1}\right)-F\left(\phi_{2}\right)\right\| \leq L\left\|\phi_{1}-\phi_{2}\right\|, \phi_{1}, \phi_{2} \in C .
$$

The history function $x_{t} \in C$ is defined by

$$
x_{t}(\theta)=x(t+\theta),-r \leq \theta \leq 0 .
$$

Definition 2.1. A continuous function $x:[-r, T] \rightarrow X, T>0$, is called an integral solution of (2.1) if

(i) $\int_{0}^{t} x(s) d s \in D(A), t \geq 0$.

(ii) $x(t)=\phi(0)+A \int_{0}^{t} x(s) d s+\int_{0}^{t} F\left(x_{s}\right) d s$.

(iii) $x_{0}=\phi$.

Definition 2.2. A family $\{S(t)\}_{t \geq 0}$ of linear operators on $X$ is called an integral semigroup on $X$ if

(i) $S(0)=0$.

(ii) $t \longmapsto S(t)$ is strongly continuous.

(iii) $S(s) S(t)=\int_{0}^{s}[S(t+r)-S(r)] d r, t, s \geq 0$.

Definition 2.3. An operator $A$ is said to be a generator of $\{S(t)\}_{t \geq 0}$ if there exists $\omega \in \mathbb{R}$ such that

(i) $(\omega, \infty) \subset \varphi(A)$.

(ii) $R(\lambda, A)=(\lambda I-A)^{-1}=\lambda \int_{0}^{\infty} e^{-\lambda t} S(t) d t, \lambda>\omega$.

Kellerman and Hieber [8] obtained the following.

Lemma 2.4. $A$ is a generator of a locally Lipschitz continuous integrated semigroup if and only if $A$ is a Hille-Yosida operator.

Let $A_{0}$ be a part of $A$ in $\overline{D(A)}$ defined by

$$
A_{0}=A \text { on } D\left(A_{0}\right)=\{x \in D(A): A x \in \overline{D(A)}\} .
$$

Lemma 2.5. [10] $A_{0}$ generates a $C_{0}$ semigroup $\left\{T_{0}(t)\right\}_{t \geq 0}$ on $\overline{D(A)}$.

Note that

$$
S(t) x=\lim _{\lambda \rightarrow \infty} \int_{0}^{t} T_{0}(s) \lambda(\lambda I-A)^{-1} x d s .
$$

In view of (2.2), the integral solution of (2.1) can be defined as the following. 
Definition 2.6. Let $T>0$ and $\phi \in C$ with $\phi(0) \in \overline{D(A)}$. A function $x:[-r, T] \rightarrow X$ is called an integral solution of (2.1) if

$x(t)=\left\{\begin{array}{l}T_{0}(t) \phi(0)+\lim _{\lambda \rightarrow \infty} \int_{0}^{t} T_{0}(t-\tau) \lambda(\lambda I-A)^{-1} F\left(x_{\tau}\right) d \tau, 0 \leq t \leq T \\ \phi(t),-r \leq t \leq 0 .\end{array}\right.$

Adimy and Ezzinbi [1] obtained the existence of integral solution of (2.1).

Lemma 2.7. Under the conditions (H1) and (H2), (2.1) has a unique integral solution $x:[-r, \infty) \rightarrow X$.

Definition 2.8. A solution operator $U(t): \widetilde{C} \rightarrow X$ is defined by

$$
U(t) \phi=x_{t}(\cdot, \phi), t \geq 0,
$$

where $\widetilde{C}=\{\phi \in C: \phi(0) \in \overline{D(A)}\}$ and $x(\cdot, \phi)$ is an integral solution of (2.1).

Lemma 2.9. [1] $\{U(t)\}_{t \geq 0}$ is a $C_{0}$ semigroup on $\widetilde{C}$.

The following dynamical notions are from [6].

Definition 2.10. A set $A \subset X$ attracts a set $B$ under $U(t)$ if

$$
\operatorname{dist}(U(t) B, A) \rightarrow 0 \text { as } t \rightarrow \infty \text {. }
$$

Definition 2.11. A set $A \subset X$ is invariant under $U(t)$ if for all $x \in A$, the orbit $\gamma(x)=U(t) x$ is contained in $A$.

Definition 2.12. An invariant set $A \subset X$ is called a global attractor of a semigroup $\{U(t)\}_{t \geq 0}$ if $A$ is a maximal compact invariant set which attracts each bounded set $B \subset X$.

Definition 2.13. A semigroup $\{U(t)\}_{t \geq 0}$ is said to be point dissipative if there exists a bounded set $B \subset X$ that attracts each point of $X$ under $U(t)$.

You and Yuan [11] proved the existence of a global attractor of (2.1) by using the following.

Lemma 2.14. [2] If there exists $t_{0}>0$ such that $U(t)$ is compact for $t>t_{0}$, and $U(t)$ is point dissipative, then there exists a nonempty global attractor in $X$. 
Now, we consider the equation $(r=0$ in $(2.1))$

$$
\left\{\begin{array}{l}
x^{\prime}(t)=A x(t)+F(x(t)), t \geq 0, \\
x(0)=x_{0} \in X .
\end{array}\right.
$$

with the assumptions

(H1) $A: D(A) \subset X \rightarrow X$ is a Hille-Yosida operator.

(H2) $F: X \rightarrow X$ is Lipshitz continuous with Lipschitz constant $L$. Then (2.4) has a unique integral solution $x:[0, \infty) \rightarrow X$ defined by

$$
x(t)=T_{0}(t) x_{0}+\lim _{\lambda \rightarrow \infty} \int_{0}^{t} T_{0}(t-\tau) \lambda(\lambda I-A)^{-1} F(x(\tau)) d \tau, t \geq 0
$$

by (2.3). Define $U(t): X \rightarrow X$ by

$$
U(t) x_{0}=x(t) .
$$

Then, by Lemma $2.9,\{U(t)\}_{t \geq 0}$ is a $C_{0}$ semigroup on $X$. Furthermore, we assume that

(H3) $\left\|T_{0}(t)\right\| \leq e^{-\alpha t}$ for some constant $\alpha>0$.

(H4) $T_{0}(t), t \geq 0$, is compact.

We need the following generalized Gronwall's inequality to obtain the estimation of the integral solution for (2.4).

LEMMA 2.15. [11] If

$$
x(t) \leq h(t)+\int_{t_{0}}^{t} k(s) x(s) d s, t_{0} \leq t<T,
$$

where all the functions involved are continuous on $\left[t_{0}, T\right), T \leq \infty$, and $k(t) \geq 0$, then $x(t)$ satisfies

$$
x(t) \leq h(t)+\int_{t_{0}}^{t} h(s) k(s) e^{\int_{s}^{t} k(u) d u} d s, t_{0} \leq t<T .
$$

Theorem 2.16. Assume that (H1)-(H3) hold true. Then we have

$$
\|x(t)\| \leq \frac{c_{1}}{\alpha-L}+\left(\|x(0)\|-\frac{c_{1}}{\alpha-L}\right) e^{(L-\alpha) t}, t \geq 0,
$$

where $c_{1}=\|F(0)\|$.

Proof. Note that

$$
\begin{aligned}
\|F(x)\| & =\|F(x)-F(0)+F(0)\| \leq\|F(0)\|+\|F(x)-F(0)\| \\
& \leq c_{1}+L\|x\| .
\end{aligned}
$$


Then, from (2.5), we have

$$
\begin{aligned}
&\|x(t)\| \\
& \leq\left\|T_{0}(t)\right\|\left\|x_{0}\right\|+\lim _{\lambda \rightarrow \infty} \int_{0}^{t}\left\|T_{0}(t-\tau)\right\|\left\|\lambda(\lambda I-A)^{-1}\right\|\|F(x(\tau))\| d \tau \\
& \leq e^{-\alpha t}\left\|x_{0}\right\|+\lim _{\lambda \rightarrow \infty} \int_{0}^{t} c_{1} e^{-\alpha(t-\tau)} \frac{\lambda}{\lambda-\omega} d \tau \\
&+\lim _{\lambda \rightarrow \infty} \int_{0}^{t} e^{-\alpha(t-\tau)} \frac{\lambda}{\lambda-\omega} L\|x(\tau)\| d \tau \\
& \leq e^{-\alpha t}\left\|x_{0}\right\|+c_{1} e^{-\alpha t} \int_{0}^{t} e^{\alpha \tau} d \tau+L e^{-\alpha t} \int_{0}^{t} e^{\alpha \tau}\|x(\tau)\| d \tau \\
& \leq e^{-\alpha t}\left[\left\|x_{0}\right\|+\frac{c_{1}}{\alpha}\left(1-e^{-\alpha t}\right)\right]+L e^{-\alpha t} \int_{0}^{t} e^{\alpha \tau}\|x(\tau)\| d \tau .
\end{aligned}
$$

Thus

$$
e^{\alpha t}\|x(t)\| \leq\left\|x_{0}\right\|+\frac{c_{1}}{\alpha}\left(e^{\alpha t}-1\right)+L \int_{0}^{t} e^{\alpha \tau}\|x(\tau)\| d \tau .
$$

In view of the generalized Gronwall's inequality (Lemma 2.15) we have

$$
\begin{aligned}
e^{\alpha t}\|x(t)\| \leq & \left\|x_{0}\right\|+\frac{c_{1}}{\alpha}\left(e^{\alpha t}-1\right)+L \int_{0}^{t}\left[\left\|x_{0}\right\|+\frac{c_{1}}{\alpha}\left(e^{\alpha s}-1\right)\right] e^{\int_{s}^{t} L d \tau} d s \\
= & \left\|x_{0}\right\|+\frac{c_{1}}{\alpha}\left(e^{\alpha t}-1\right)+L\left\|x_{0}\right\| e^{L t} \int_{0}^{t} e^{-L s} d s \\
& +\frac{c_{1} L}{\alpha} e^{L t}\left(\int_{0}^{t} e^{(\alpha-L) s} d s-\int_{0}^{t} e^{-L s} d s\right) \\
= & \left\|x_{0}\right\|+\frac{c_{1}}{\alpha}\left(e^{\alpha t}-1\right)+\left\|x_{0}\right\|\left(e^{L t}-1\right) \\
& +\frac{c_{1} L}{\alpha(\alpha-L)}\left(e^{\alpha t}-e^{L t}\right)-\frac{c_{1}}{\alpha}\left(e^{L t}-1\right) \\
= & \left(\frac{c_{1}}{\alpha}+\frac{c_{1} L}{\alpha(\alpha-L)}\right) e^{\alpha t}+\left(\left\|x_{0}\right\|-\frac{c_{1} L}{\alpha(\alpha-L)}-\frac{c_{1}}{\alpha}\right) e^{L t} \\
= & \frac{c_{1}}{\alpha-L} e^{\alpha t}+\left(\left\|x_{0}\right\|-\frac{c_{1}}{\alpha-L}\right) e^{L t}, t \geq 0 .
\end{aligned}
$$

Hence we obtain the estimation (2.6). This completes the proof.

Corollary 2.17. Under the hypotheses of Theorem 2.16, $U(t), t \geq$ 0 , is point dissipative if $\alpha \neq L$. 
Proof. There exists $t_{0}>0$ such that

$$
\|x(t)\| \leq \frac{c_{1}}{\alpha-L}+1=k, t \geq t_{0}
$$

by Theorem 2.16. It follows that the ball $B(0, k)$ attracts each point of $X$.

Theorem 2.18. Assume that (H1)-(H4) hold true. Then $U(t)$ is compact for $t>0$.

Proof. Let $\left(\phi_{n}\right)$ be a bounded sequence in $\widetilde{C}(\mathbb{R}, X)=\{x \in C(\mathbb{R}, X)$ : $x(0) \in \overline{D(A)}\}$. We show that $\left\{U(t) \phi_{n}: n \in \mathbb{N}\right\}$ is relatively compact in $\widetilde{C}(\mathbb{R}, X)$. Note that

$$
\begin{aligned}
& \left(U(t) \phi_{n}\right)(\theta) \\
& =T_{0}(t+\theta) \phi_{n}(0)+\lim _{\lambda \rightarrow \infty} \int_{0}^{t+\theta} T_{0}(t+\theta-\tau) \lambda(\lambda I-A)^{-1} F(x(\tau)) d \tau .
\end{aligned}
$$

Since $\left(\phi_{n}\right)$ is bounded and $T_{0}(t)$ is compact by $(\mathrm{H} 4),\left\{T_{0}(t+\theta) \phi_{n}(0)\right.$ : $n \in \mathbb{N}\}$ is relatively compact. We have, for small $\varepsilon>0$,

$$
\begin{aligned}
\lim _{\lambda \rightarrow \infty} \int_{0}^{t+\theta} T_{0}(t+\theta-\tau) \lambda(\lambda I-A)^{-1} F(x(\tau)) d \tau \\
=T_{0}(\varepsilon) \lim _{\lambda \rightarrow \infty} \int_{0}^{t+\theta-\varepsilon} T_{0}(t+\theta-\tau-\varepsilon) \lambda(\lambda I-A)^{-1} F(x(\tau)) d \tau \\
\quad+\lim _{\lambda \rightarrow \infty} \int_{t+\theta-\varepsilon}^{t+\theta} T_{0}(t+\theta-\tau) \lambda(\lambda I-A)^{-1} F(x(\tau)) d \tau .
\end{aligned}
$$

Note that $\sup \|x(t)\|<\infty$ by Theorem 2.16 and $\|F(x)\| \leq\|F(0)\|$ $+L\|x\|$ by (H2). Thus there exist $M_{1}>0$ and $M_{2}>0$ such that

$$
\left\|\lim _{\lambda \rightarrow \infty} \int_{t+\theta-\varepsilon}^{t+\theta} T_{0}(t+\theta-\tau) \lambda(\lambda I-A)^{-1} F(x(\tau)) d \tau\right\| \leq M_{2}
$$

and

$$
\left\|\lim _{\lambda \rightarrow \infty} \int_{0}^{t+\theta-\varepsilon} T_{0}(t+\theta-\tau-\varepsilon) \lambda(\lambda I-A)^{-1} F(x(\tau)) d \tau\right\| \leq M_{1} \varepsilon .
$$

This implies that

$$
T_{0}(\varepsilon)\left\{\lim _{\lambda \rightarrow \infty} \int_{0}^{t+\theta-\varepsilon} T_{0}(t+\theta-\tau-\varepsilon) \lambda(\lambda I-A)^{-1} F(x(\tau)) d \tau\right\} \subset K_{\varepsilon}
$$

for some compact set $K_{\varepsilon} \subset X$. Therefore $\left\{\left(U(t) \phi_{n}\right)(\theta): n \in \mathbb{N}\right\}$ is relatively compact. Also, we can show that the family $\left\{U(t) \phi_{n}: \phi_{n} \in\right.$ 
$\widetilde{C}(\mathbb{R}, X)\}$ is equicontinuous. Therefore $U(t)$ is compact for $t>0$. This completes the proof.

Now, we obtain the following result by Theorem 2.16, Corollary 2.17 and Theorem 2.18.

Theorem 2.19. Assume that (H1)-(H4) hold true. Then (2.4) has a nonempty global attractor provided $\alpha>L$.

\section{References}

[1] M. Adimy and K. Ezzinbi, Local existence and linearized stability for partial functional differential equations, Dynam. Syst. Appl. 7 (1998), 389-403.

[2] J. Billotti and J. P. LaSalle, Dissipative periodic processes, Bull. Amer. Math. Soc. 77 (1971), 1082-1088.

[3] H. Bouzahir and K. Ezzinbi, Global attractor for a class of partial functional differential equations with infinite delay, in : T. Faria, P. Freitas(Eds.), Topics in Functional Difference Equations, Fields Inst. Commun., vol. 29, Amer. Math. Soc., Providence, RI, 2001, 63-71.

[4] H. Bouzahir, H. You, and R. Yuan, Global attractor for some partial functional differential equations with infinite delay, Funckcial. Ekvac. 54 (2011), 139-156.

[5] T. Caraballo, P. Marin-Rubio, and J. Valero, Attractors for differential equations with unbounded delays, J. Differential Equations 239 (2007), 311-342.

[6] J. Hale, Asymptotic Behavior of Dissipative Systems, Math. Surveys and Monographs, vol. 25, Amer. Math. Soc., Providence, RI, 1988.

[7] J. Hale and J. Kato, Phase space for retarded equations with infinite delay, Funkcial. Ekvac. 21 (1978), 11-41.

[8] H. Kellermann and M. Hieber, Integrated semigroup, J. Funct. Anal. 15 (1989), $160-180$.

[9] A. Pazy, Semigroup of Linear Operators and Applications to Partial Differential Equations, Appl. Math. Sci., vol. 44, Springer-Verlag, New York, 1983.

[10] H. R. Thieme, Semiflows generated by Lipschitz perturbations of non-densely defined operators, Differential Integral Equations 3 (1990), 1035-1066.

[11] H. You and R. Yuan, Global attractor for some partial functional differential equations with finite delay, Nonlinear Analysis 72 (2010), 3566-3574.

[12] M. C. Zelatti, On the theory of global attractors and Lyapunov functionals, Set-Valued Var. Anal. 21 (2013), 127-149. 
Department of Mathematics

Chungnam National University

Daejeon 305-764, Republic of Korea

E-mail: sgchoi@cnu.ac.kr

$* *$

Department of Mathematics

Chungnam National University

Daejeon 305-764, Republic of Korea

E-mail: hhchang@cnu.ac.kr

$* * *$

Department of Mathematics Chungnam National University Daejeon 305-764, Republic of Korea

E-mail: njkoo@cnu.ac.kr

$* * * *$

Department of Mathematics

Chungnam National University

Daejeon 305-764, Republic of Korea

E-mail: cmyun@cnu.ac.kr 